\title{
A Model Experiment on the Gas Entrainment with Pouring Liquid Steel Stream*
}

\author{
By Katsuyoshi IWATA,** Takao $\mathrm{CHOH}^{* * *}$ \\ and Michio INOUYE***
}

\section{Synopsis}

In order to understand the air oxidation of molten steel during teeming, the model experiments on the gas entrainment of pouring stream were carried out by using water, ethanol, glycerin aqua solution and liquid tin.

The breakup length of pouring stream depends on the shape of nozzle, the pouring rate of liquid and the flow of liquid in the tank.

It was found that, in the range of the present experiment, the amplitude of disturbance at the nozzle exit, $\varepsilon_{0}$, decreases with the decrease of nozzle length and pouring rate as indicated by the following equation.

$$
\ln \left(a_{0} / \varepsilon_{0}\right)=44.3\left(l_{0} / D_{0}\right)^{-0.75}(R e)^{-0.1}
$$

The rates of gas entrainment by the pouring stream increase with increasing height of teeming and rate of teeming.

It is confirmed that the rate of gas entrainment can be represented by a model assuming that the gas entrainment does not occur under the true cylindrical stream but arises by the cavity produced by the collision between the bath surface and the stream surface protuberances.

Consequently, taking the cavity radius $R_{c}$ into account, the rates of gas entrainment can be described for all kinds of liquid stream as follows;

$$
V_{g} / V_{l}=0.02\left\{\left(R_{c}-a\right) / a_{0}\right\}^{3} .
$$

\section{Introduction}

Flow behaviors of molten steel during teeming are generally difficult to analyze because of their strong dependence on those factors such as the dimension of nozzle, the reaction with gas phase, the depth of liquid steel in ladle and so on. For the pouring processes, however, it is important to understand the characteristics of liquid steel stream. It is well known that the liquid steel absorbs oxygen from the surrounding atmosphere during teeming, resulting in the oxidation of alloying elements or the increase of nonmetallic inclusions. It is also probable that most of oxygen absorption comes from the air bubbles entrained during teeming rather than the transfer through the stream surface or the free surface of molten steel.

In the present work, a model experiment is performed to obtain the relationship between the rate of gas entrainment and the behavior of teeming stream or the mechanism of gas entrainment.

\section{Stability of Liquid Stream}

\section{Previous Works}

Many studies were made on the stability of liquid stream such as the experimental studies ${ }^{1-4)}$ and the application of stability theory to the horizontal ${ }^{5-7)}$ or the vertical liquid stream. ${ }^{4,8,9}$ )

The investigations on the breakup of stream were firstly conducted by Savart ${ }^{10}$ ) and Rayleich,5) who deduced the stability theory of nonviscous liquid stream. Then, Weber ${ }^{6)}$ made a quantitative description of the stability of a viscous stream on the basis of Rayleich's theory. On the other hand, Ohnesorge ${ }^{11)}$ developed a classification that all streams can be classified into three distinct categories by the flow rate as shown in Fig. 1. The stream in region (I) breaks due to the growth of symmetrical disturbances. In region (II), the aerodynamic forces acting on the surface of a stream tend to propagate asymmetrical disturbances and then the breakup of stream occurs by wave vibration. The stream in region (III) presents the atomization into a cloud of small droplets. Massard and Lange ${ }^{12,13)}$ divided the region (II) into three subdivisions $A, B$ and $C$ and pointed out that, although the stability theory could be applicable for the stream in the region (II)A, a stream over this region is represented by an empirical relationship.

On the other hand, Takahashi and Kitamura ${ }^{8)}$ expressed the value of $l_{z}$ in the region (I) by Eq. (1) from the analyzed results on the vertical stream by Duda and Vrentas ${ }^{14)}$ and Kurabayashi, ${ }^{15)}$ and also the consideration shown by Ziabicki and Krozer. ${ }^{16)}$

$$
l_{z} / D_{0}=18 W e^{4 / 7} \mathrm{Fr}^{-1 / 7}
$$

In the present work, considering that the gas entrainment by teeming stream depends on the surface disturbances of stream or on the values of breakup length $l_{z}$, the stability of vertical stream at a higher flow rate is analyzed.

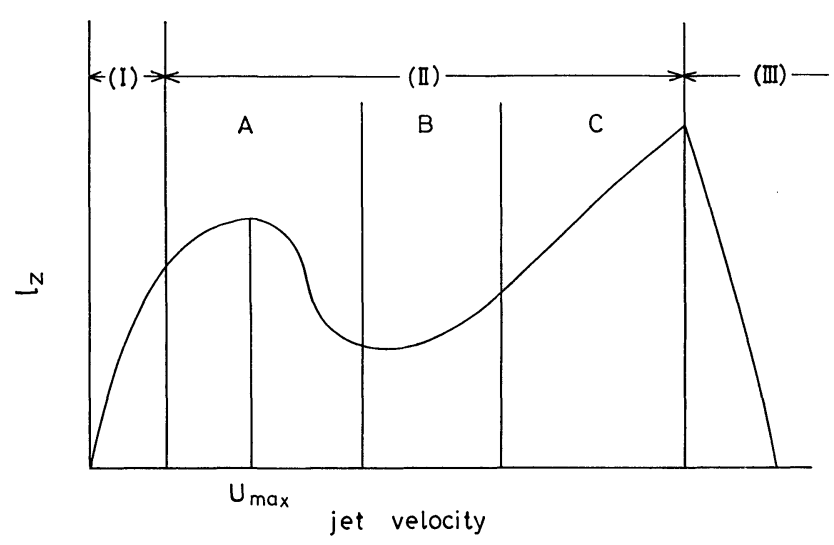

Fig. 1. General shape of breakup curve.

* Presented to the 99th ISIJ Meeting, April 1980, at The University of Tokyo in Tokyo. Manuscript received March $10,1982$. (C) 1983 ISIJ

** Formerly Faculty of Engineering, Nagoya University. Now at Wakayama Steel Works, Sumitomo Metal Industries, Ltd., Minato, Wakayama 641.

*** Faculty of Engineering, Nagoya University, Furo-cho, Chikusa-ku, Nagoya 464. 


\section{Theory of Stability of Liquid Stream}

As well known, the liquid stream surface shows some disturbances having various wave lengths and various growth rates. Among them, a disturbance of the particular wave length $\left(\lambda_{\max }\right)$ grows and then the stream begins to break up. Previously Levich ${ }^{7)}$ presented the following relationships for the growth rate $\left(W_{\max }\right)$ and the wave number $\left(k_{\max }\right)$ of the horizontal liquid stream*:

(A) Liquid stream having the symmetrical disturbance at low flow rate:

$$
\begin{aligned}
W_{\max } & =\left\{\sigma /\left(8 \rho a^{3}\right)\right\}^{1 / 2} \\
k_{\max } & \left.=2 \pi / \lambda_{\max }=1 / \sqrt{2} a\right)
\end{aligned}
$$

(B) Liquid stream depending on aerodynamic forces at higher flow rate:

$$
\begin{aligned}
W_{\max } & =\left\{\rho_{g} U_{0}^{2} /\left(6 \rho a^{2}\right)+\sigma /\left(8 \rho a^{3}\right)\right\}^{1 / 2} . \\
k_{\max } & =2 \pi / \lambda_{\max } \simeq 0.75 / a \ldots \ldots \ldots \ldots \ldots \ldots
\end{aligned}
$$

(C) Liquid stream at high flow rate

$$
\begin{aligned}
W_{\max } & \simeq\left(\rho_{g} / 8 \rho\right)^{1 / 2} \cdot\left(U_{0} / a\right) \\
k_{\max } & \simeq 1.56 / a
\end{aligned}
$$

In the present work, the vertical liquid streams were analyzed on the basis of above relationships in (A) and (B). For the case of (A), Takahashi and Kitamura ${ }^{8)}$ have already analyzed by the following method. As for the stability of a vertical laminar liquid stream, Kurabayashi ${ }^{15)}$ obtained the following equation.

$$
2 g z / U_{0}^{2}+1=\left(a_{0} / a\right)^{4}+8\left\{a_{0} / a-1\right\} / W e_{0}
$$

Assuming that Weber number at the nozzle exit is large enough or $a \simeq a_{0}$, the radius $a$ of liquid stream at the distance $z$ can be expressed by Eqs. (9) and (10) from Eq. (8).

$$
\begin{aligned}
& a=a_{0} \xi^{2} \ldots \ldots \ldots \ldots \ldots \ldots \\
& \xi=\left(2 g z / U_{0}^{2}+1\right)^{1 / 8} .
\end{aligned}
$$

Further, using the equation of continuity, the velocity at the distance $z$ can be described by Eq. (11).

$$
U=U_{0} \xi^{4}
$$

The amplitude $\varepsilon$ of disturbance at $z$ is given by Eq. (12).

$$
\varepsilon=\varepsilon_{0} \exp \left\{\int_{0}^{z}\left(W_{\max } / U\right) d z\right\}
$$

Substituting Eqs. (2), (9) and (11) into Eq. (12) and integrating, Eq. (12) becomes

$$
\begin{aligned}
\varepsilon & =\varepsilon_{0} \exp \left(\xi^{7}-1\right) /(7 \alpha) \\
\alpha & =\left\{g^{2} \rho a_{0}^{3} /\left(2 U_{0}^{2} \sigma\right)\right\}^{1 / 2}
\end{aligned}
$$

When the amplitude is equal to the stream radius, that is $a\left(l_{z}\right)=\varepsilon\left(l_{z}\right)$, the stream breaks up. Now substituting Eqs. (9) and (13) into this relationship and taking $\xi\left(l_{z}\right)=X$, Eq. (14) can be deduced.

$$
\left(X^{7}-1\right) /(7 \alpha)=\ln \left(a_{0} / \varepsilon_{0}\right)+\ln X^{-2} .
$$

Then assuming that the effects of stream velocity on the values of $\ln X^{-2}$ and $\ln \left(a_{0} / \varepsilon_{0}\right)$ are negligible and $2 g z / U_{0}^{2}$ is sufficiently large compared to unity, Eq. (15) expressed by Takahashi and Kitamura ${ }^{8)}$ can be obtained.

$$
\left.\begin{array}{l}
l_{z} / D_{0}=A_{0} F r^{-1 / 7} W e^{4 / 7} \\
A_{0}=(1 / 2)\left\{(7 / 4)\left(\ln \left(a_{0} / \varepsilon_{0}\right)+\ln X^{-2}\right)\right\}^{8 / 7}
\end{array}\right\} \cdots \cdots .
$$

On the other hand, when the stream velocity becomes higher and the surface of liquid stream is affected by aerodynamic force of surrounding gas as in the case of (B), the amplitude $\varepsilon$ of disturbances at $z$ is given by Eq. (16) from Eqs. (4), (9), (11) and (12).

$$
\varepsilon=\varepsilon_{0} \exp \left[\int_{0}^{\xi} 4 \operatorname{Fr}\left\{(2 / 3)\left(\rho_{g} / \rho\right) \xi^{6}+W e^{-1}\right\}^{1 / 2} \xi^{6} d \xi\right] \ldots
$$

It is found that, in the range of $\xi$ from 1.0 to 1.3 , the values of

$$
\left\{(2 / 3)\left(\rho_{g} / \rho\right) \xi^{6}+W e^{-1}\right\}^{1 / 2}
$$

are almost independent of $\xi$, taking $W e$ as a parameter. Also, $\xi^{\mathbf{6}}$ in the above relation \{\} could be approximately taken as 2, then Eq. (16) can be represented by Eq. (17).

$$
\left.\begin{array}{l}
\varepsilon=\varepsilon_{0} \exp \left\{1 /(7 \beta)\left(\xi^{7}-1\right)\right\} \\
1 / \beta=4 \operatorname{Fr}\left\{(4 / 3)\left(\rho_{g} / \rho\right)+W e^{-1}\right\}^{1 / 2}
\end{array}\right\}
$$

An error due to this approximation may be nearly $20 \%$ for such a condition as the teeming of liquid steel, $W e=10000$ and $\xi=1.0 \sim 1.2$. Then substituting Eqs. (17) and (9) into the breakup condition $a\left(l_{z}\right)=\varepsilon\left(l_{z}\right)$, the breakup length is given by the equation

$$
l_{z} / D_{0}=A_{0} F r^{-1 / 7}\left\{(4 / 3)\left(\rho_{g} / \rho\right)+W e^{-1}\right\}^{-4 / 7}
$$

Furthermore, by an approximation as

$$
\left\{(4 / 3)\left(\rho_{g} / \rho\right)+W e^{-1}\right\}^{1 / 2}=r W e^{-s},
$$

Eq. (18) can be simplified to Eq. (19) that is not given by Takahashi et al. However Eq. (19) is similar to Eq. (15).

$$
\left.\begin{array}{l}
l_{z} / D_{0}=A F r^{-1 / 7} W e^{8 s / 7} \\
A=A_{0} r^{-8 / 7}
\end{array}\right\}
$$

* Levich ${ }^{7)}$ expressed the growth rate of disturbance on the stream surface by the following relation:

$$
W=\left[-\frac{\rho_{g} \cdot(k a)^{4} \cdot U_{0}^{2}}{2 a^{2}}\left\{\ln \left(\frac{k a}{2}\right)\right\}+\frac{(k a)^{2}}{2 \rho a^{3}}\left\{1-(k a)^{2}\right\}\right]^{1 / 2}
$$

At low flow velocity, the first term of above equation can be neglected, and the value of $W$ reaches a maximum at $k a=1 / \sqrt{2}$ as given by Eqs. (2) and (3), where those values of $W, k$ and $\lambda$ are represented at maximum by $W_{\max }, k_{\max }$ and $\lambda_{\max }$, respectively. On the other hand, Eqs. (6) and (7) are established when the flow velocity is high enough for neglecting the second term of above relation and, under the intermediate flow velocity, Eqs. (4) and (5) are deduced by taking both terms into account. 
The values of $r$ and $s$ in Eq. (19) are determined experimentally. If an index $8 s / 7$ of Weber number is given, the relation which is valid for the present work can be decided from Eqs. (13) and (17) for the amplitude of surface disturbances and from Eqs. (15) and (19) for the breakup length.

\section{Experimental Methods}

In the present work, three methods (a), (b) and (c) were used for the measurements of breakup lengths $\left(l_{z}\right)$ of water, ethanol, glycerin aqua solutions (12.5 and $25 \%$ glycerin) and liquid tin.

(a) System for Constant Teeming Rate

The experimental apparatus is shown in Fig. 2(A). The velocity of water at the nozzle exit can be controlled by changing the position of water tank (1) from which a part of supplied water flows out in order to maintain the water level at a constant value. The breakup length $\left(l_{z}\right)$ was firstly measured at the position (3) by taking several $35 \mathrm{~mm}$ still photographs of teeming stream of nearly $50 \mathrm{~cm}$ length at the breakup region. Also, the values of $l_{z}$ and $\varepsilon$ were estimated from the fluctuation of stream diameter found in the cinematograph pictures taken at several positions above the breakup region. The dimensions of glass nozzle are as follows; internal diameters: $0.6,0.8,1.0$ $\mathrm{cm}$ and length: $3 \mathrm{~cm}$.

(b) System for the Teeming Rate Changing with Time

In this case, the apparatus shown by Fig. 2(B) was used. The values of $l_{z}$ were determined by the photographs taken at the time when the water level in the tank (4) reaches certain given depths. The velocity at the nozzle exit was calculated by Eq. (20) deduced from the relationship, $U_{0}=\sqrt{2 g h}$, where $h$ was obtained by integrating $d h / d t=-\left(a_{0} / R_{1}\right)^{2} \sqrt{2 g h{ }^{*}}$

$$
U_{0}=\sqrt{2 g}\left\{\sqrt{h_{i}+l_{0}}-\left(a_{0} / R_{1}\right)^{2} \cdot \sqrt{g / 2} \cdot t\right\}
$$

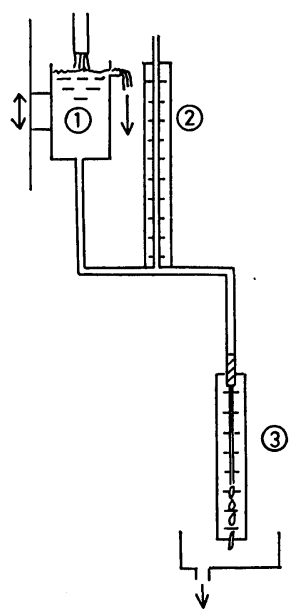

A

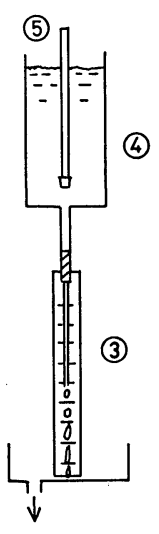

B
Fig. 2. Experimental apparatus for cold model.

\section{(c) System of Liquid Tin}

About $700 \mathrm{~g}$ tin was inductively melted under argon atmosphere in a graphite crucible equipped with a stopper and after maintaining at $350^{\circ} \mathrm{C}$ for about ten minutes, liquid tin was teemed in a mold by raising the stopper. Then the values of $l_{z}$ were determined from the cinematograph pictures of the stream. The velocity at the exit in this system was also calculated by Eq. (20). The diameters of nozzle were 0.5 and $0.7 \mathrm{~cm}$.

\section{Experimental Results and Discussions \\ 1. Breakup Length $l_{z}$}

Figure 3 shows the breakup length of water at the constant flow velocity by method (a), and Fig. 4 indicates that of ethanol under the velocity changing with

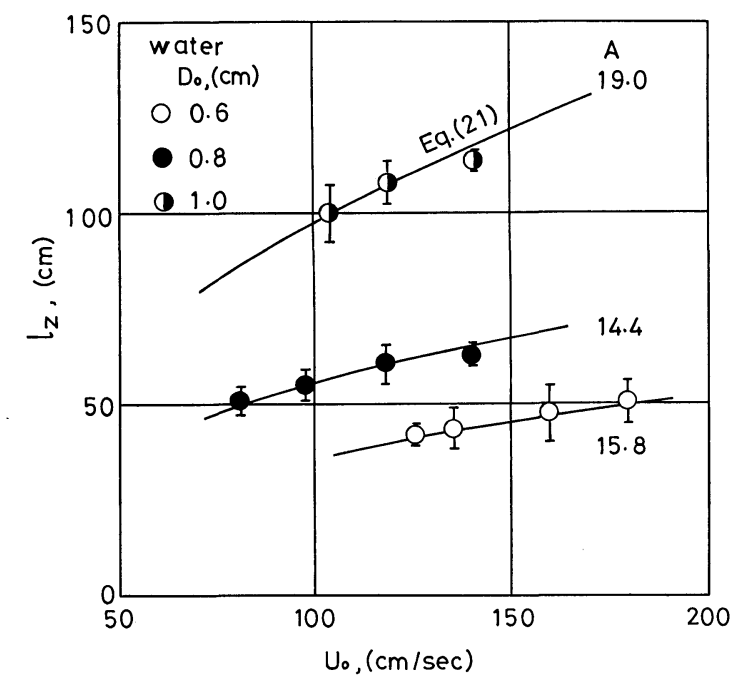

Fig. 3. Breakup length of water stream. Curves are represented by Eq. (21).

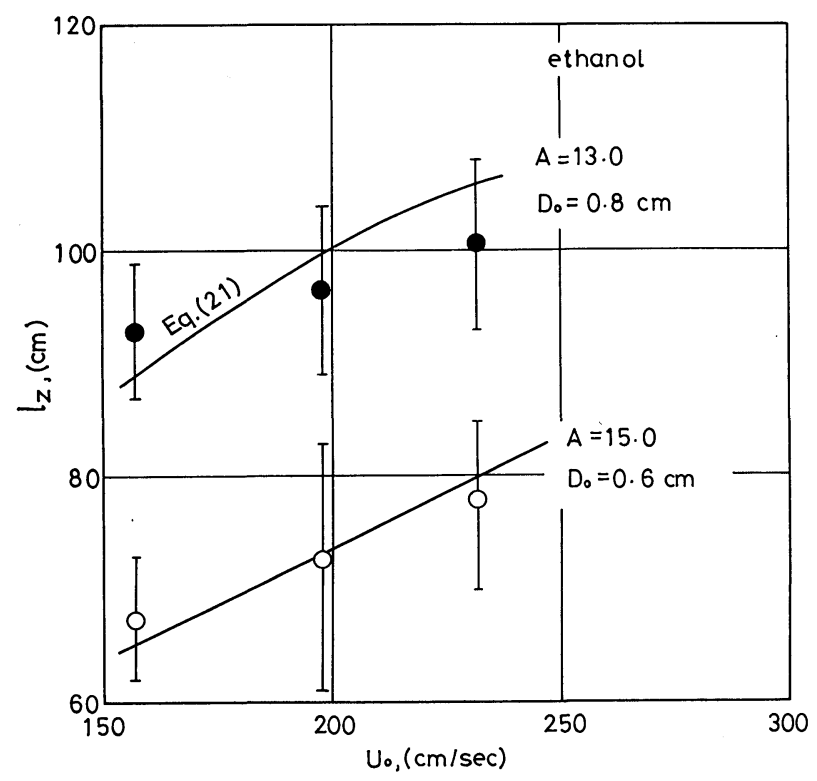

Fig. 4. Breakup length of ethanol stream. Curves are represented by Eq. (21).

* In general, the flow velocity at the nozzle exit, $U_{0}$, must be expressed exactly by $U_{0}=C_{d} \sqrt{2 g h}$ where $C_{d}$ describes a discharge coefficient. The values of $C_{d}$ for glass nozzles used in the present work were in the range from 0.93 to 0.94 , and could be assumed as $C_{d}=1$. Further, as those of $C_{d}$ in the case of experiments using liquid tin decrease to the range from 0.87 to 0.89 , velocities $U_{0}$ calculated under $G_{d}=1$ were used in the present work approximately. 
time by method (b). Further, Fig. 5 gives the results for glycerin aqua solutions (12.5 and $25 \%$ glycerin) by method (b) and for liquid tin by method (c). In every case, the values of $l_{z}$ increase with increasing nozzle diameter and increasing velocity. Those results suggest that the velocities $U_{0}$ in the present work are below or near $U_{\max }$ in Fig. 1. Plotting $\left(l_{z} / D_{0}\right)$. $F^{1 / 7}$ against $W e$ in logarithmic scale in Fig. 6 for those experimental results shown by Fig. 3, it is found that they can be expressed by the linear relationships of slope 2/5. Namely, the breakup length $l_{z}$ is not proportional to $W e^{4 / 7}$ as given by Eq. (15), but to $W e^{2 / 5}$. Therefore, the amplitude of surface disturbances of stream can be expressed by Eq. (17) and the breakup length is consequently given by Eqs. (21)

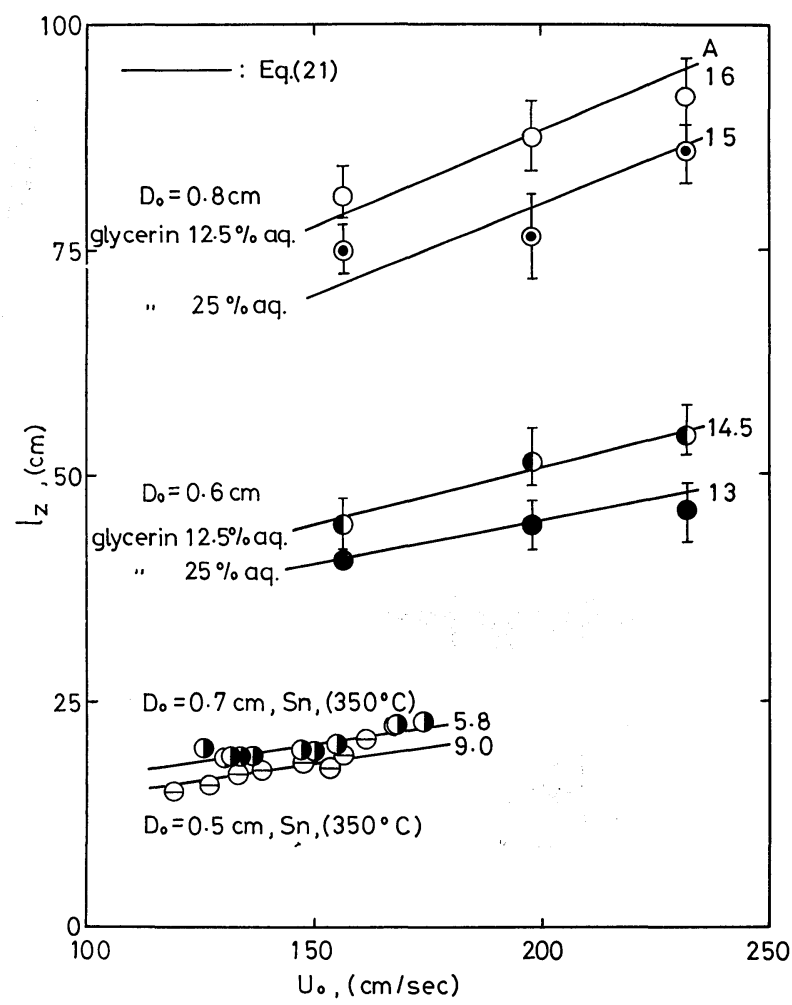

Fig. 5. Breakup length of glycerin 12.5 and $25 \%$ aqua solution and liquid tin. Curves are represented by Eq. (21).

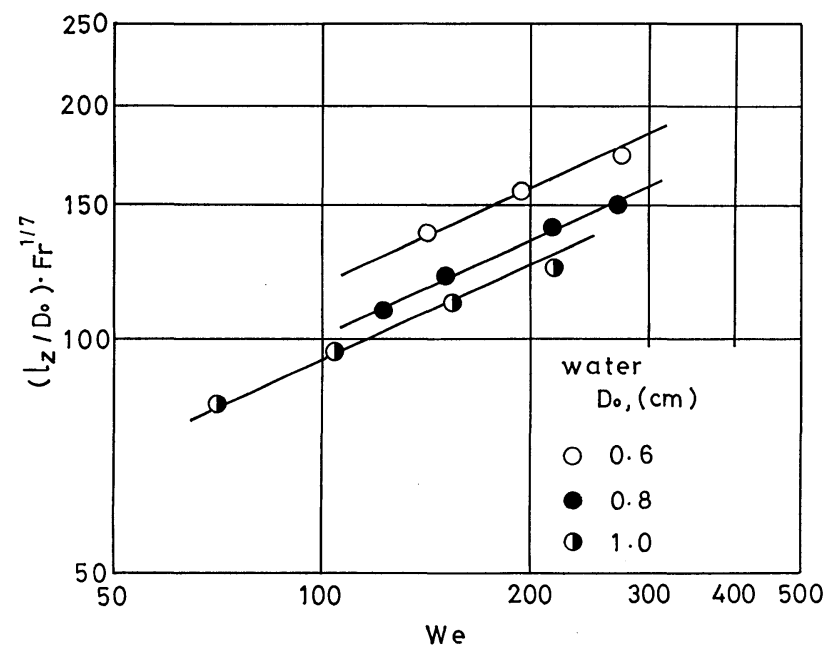

Fig. 6. Relation between $\left(l_{z} / D_{0}\right) F r^{1 / 7}$ and $W e$. and (22) from Eq. (19).

$$
\begin{aligned}
& l_{z} / D_{0}=A W e^{2 / 5} \mathrm{Fr}^{-1 / 7} \\
& A=2.21 A_{0}
\end{aligned}
$$

From those relations, the values of $r$ and $s$ become $s=0.35$ and $r=0.5$.

The breakup length $\left(l_{z}\right)$ was also obtained indirectly from the measurement of the amplitude of surface disturbances. Figure 7 shows an example of the stream diameter distribution obtained from 104 frames of the cinematograph pictures taken at the position of $25 \mathrm{~cm}$ under the nozzle exit. The mean value of this diameter distribution gives the mean radius of stream, $\bar{a}$, and also the mean deviation of this distribution gives the mean amplitude of surface disturbance of stream, $\bar{\varepsilon}$, as shown in Fig. 7. The values of $\bar{a}$ and $\bar{\varepsilon}$ obtained at several positions of the streams for nozzle diameters $D_{0}=0.6$ and $1.0 \mathrm{~cm}$ are plotted against $\xi$ in Fig. 8. It is found that, those of $\bar{\varepsilon}$ deviate from the relation of Eq. (17) in the near region of the nozzle although the values of $\bar{a}$ almost agree with Eq. (9). This is probably because of the enlargement of amplitude $\vec{\varepsilon}$ due to the various disturbances at the vicinity of the nozzle. ${ }^{17)}$ The breakup lengths $l_{z}$ obtained from the breakup condition, namely from the intersection of line $\bar{a}$ and line $\bar{\varepsilon}$, are also shown in Fig. 8, which represents that, in the case of nozzle diameter $D_{0}=0.6 \mathrm{~cm}$ (mark $\bullet$ ) where $\bar{a}$ and $\bar{\varepsilon}$ are measured to near breakup length, the value of $l_{z}$ agrees well with the result of direct measurement shown in brackets. However, the value of $l_{z}$ for the nozzle diameter $D_{0}=1.0 \mathrm{~cm}$ (mark O) becomes larger than direct measured value because the measurements are made near the nozzle at which the amplitude of disturbance $\bar{\varepsilon}$ may be enlarged. Therefore, in order to obtain the correct breakup length of stream from the amplitude of surface disturbances, the values $\bar{\varepsilon}$ must be measured at the position far from nozzle exit.

2. Effect of Fluid Flow in the Tank on the Value of $\boldsymbol{l}_{z}$

The effect of fluid flow in the tank was measured by

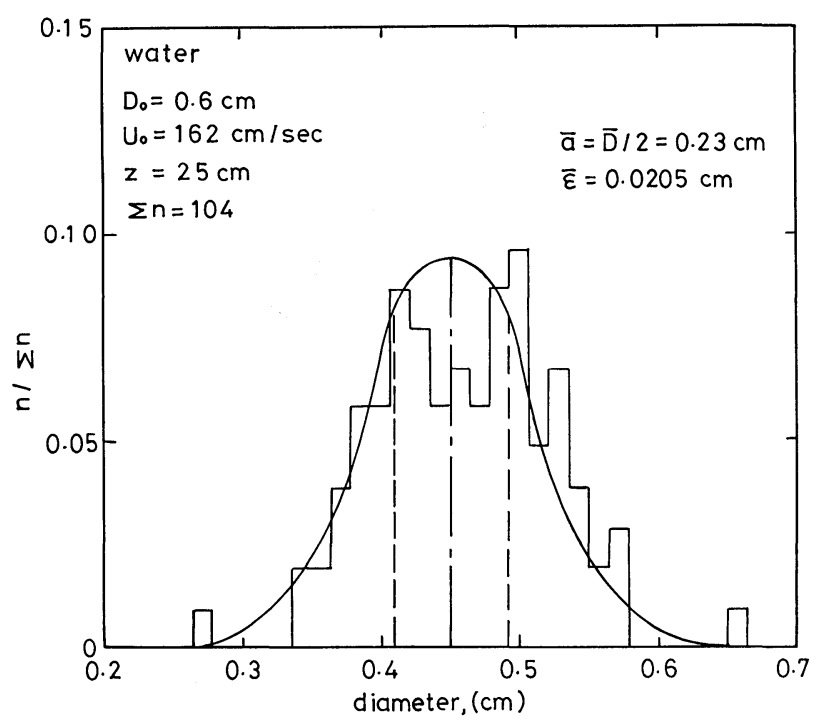

Fig. 7. Distribution curve of liquid stream diameter at $z=$ $25 \mathrm{~cm}$. 


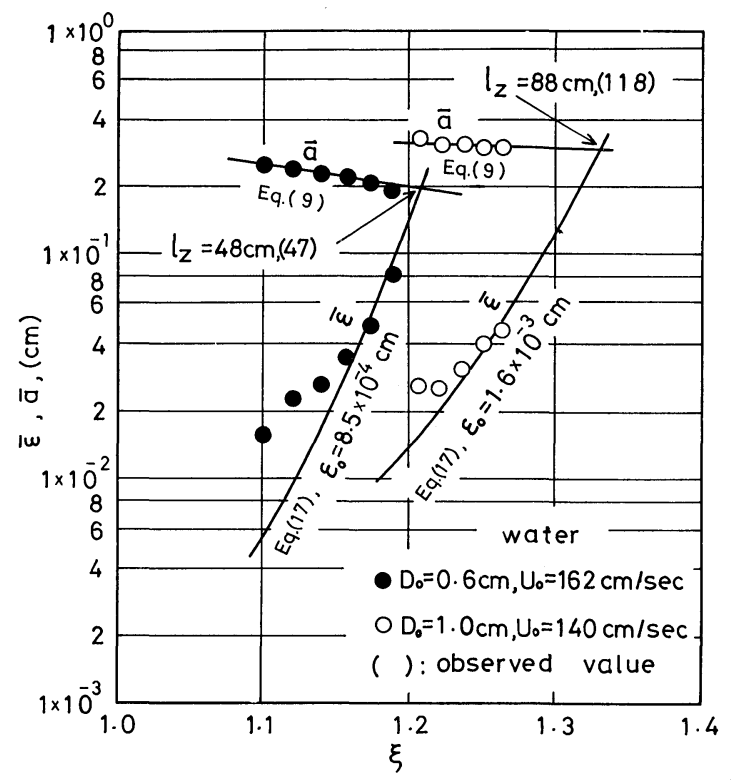

Fig. 8. Determination of breakup length.

the following method. The fluid in the tank was firstly agitated along the side wall of the tank by an agitator at $60 \mathrm{rpm}$ and after stopping the agitation, alumina powder was floated on the agitated fluid surface. Then the maximum fluid flow rate was determined from floated alumina powder and at the same time, the breakup length was measured.

The experimental results for $D_{0}=1.0 \mathrm{~cm}$ are shown in Fig. 9, indicating that the values of $l_{z}$ decrease with increasing angular velocity $\omega$. Equation (21) is also applicable for those results, selecting the available values of $A$ that decrease with increasing angular velocity. This is not due to decrease in the flow velocity at the nozzle exit, because the discharge coefficient $C_{d}$ changes only from 0.94 to 0.92 even by the increase of fluid flow in the tank from 0 to $1.66 \mathrm{rad} /$ sec, but due to the increase in the amplitude of surface disturbances on the teeming stream as observed in the experiments. The ratio, $A_{2} / A_{1}$, where $A_{1}$ and $A_{2}$ represent the value of $A$ under the still state and that under the flow state of fluid respectively, can be represented by Eq. (23), taking the dimensionless number $\rho D_{0} R_{1} \omega / \mu$, which shows the similar meaning as Reynolds number.

$$
A_{2} / A_{1}=1.0-(1 / 3000)\left(\rho D_{0} R_{1} \omega / \mu\right)
$$

\section{Initial Amplitude of Surface Disturbance, $\varepsilon_{0}$}

According to Eqs. (15) and (19), the value of initial amplitude of disturbance, $\varepsilon_{0}$, can be calculated from $A$. It was confirmed that the values of $\varepsilon_{0}$ obtained from $A$ are nearly equal to those calculated by Taylor expansion of Eq. (16) in the range of error $\pm 5 \%$, that is, those values obtained from $A$ are available for the estimation of initial amplitude of disturbance. Now, plotting $\ln \left(a_{0} / \varepsilon_{0}\right)$ obtained by Taylor expansion against the nozzle geometry $l_{0} / D_{0}$ for several fluids, a linear relationship between them is found as shown in Fig. 10. Furthermore, the relationship between the values of $\left(l_{0} / D_{0}\right)^{3 / 4} \ln \left(a_{0} / \varepsilon_{0}\right)$ and Re number is obtained by taking the slope of $-3 / 4$ for the line in

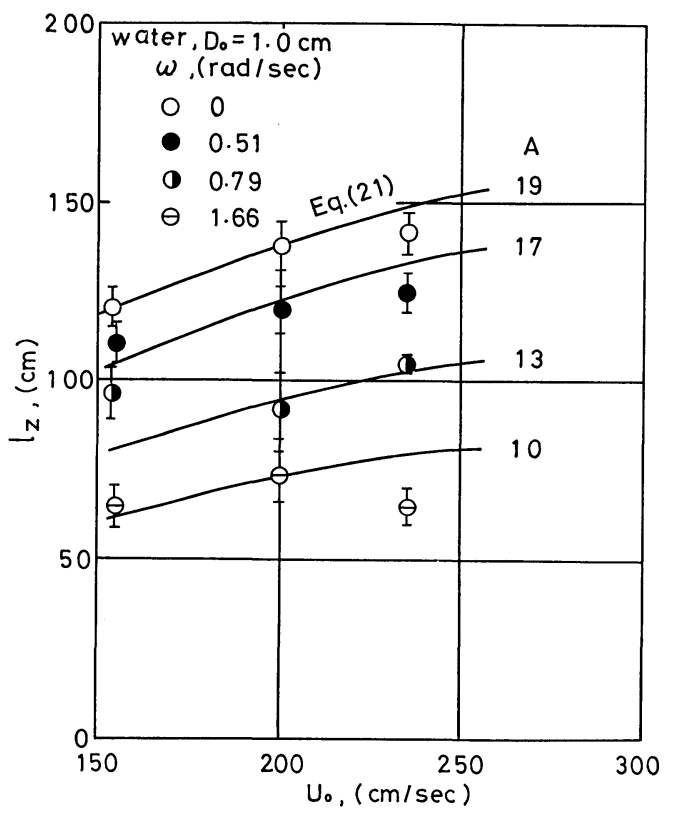

Fig. 9. Effect of angular velocity of fluid in tank on the breakup length. Curves are represented by Eq. (21).

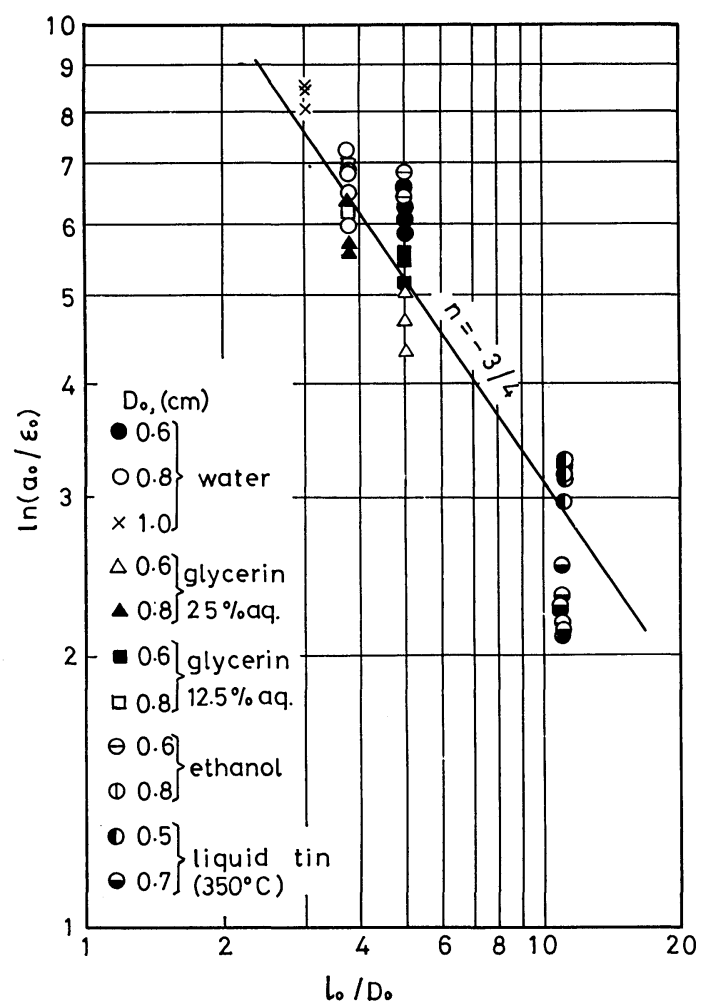

Fig. 10. Effect of the diameter and the length of nozzle on the initial amplitude of disturbance.

Fig. 10 and is expressed approximately by Eq. (24) as shown in Fig. 11.

$$
\ln \left(a_{0} / \varepsilon_{0}\right)=44.3\left(l_{0} / D_{0}\right)^{-0.75} R e^{-0.1}
$$

Accordingly, it shows that the value of $\varepsilon_{0}$ increases with increasing nozzle length, and this agrees well with those results as shown by the previous investigations. ${ }^{9,18)}$ On the other hand, although the value of $\varepsilon_{0}$ by Eq. (24) depends on $U_{0}$, the value of $A$ could be 


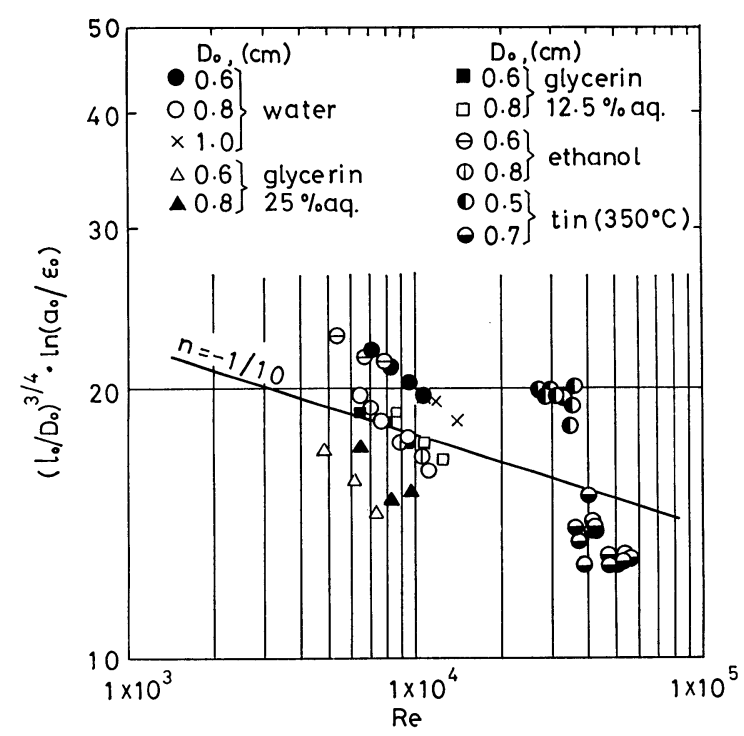

Fig. 11. Relation between $\left(l_{0} / D_{0}\right)^{3 / 4} \ln \left(a_{0} / \varepsilon_{0}\right)$ and $R e$.

taken as constant for the experimental results measured by methods (b) and (c) as shown in Figs. 4 and 5. This is probably because the change in the flow rate of present work is small.

\section{Gas Entrainment by Teeming Stream}

\section{Previous Investigations}

Yakovlev $^{19)}$ and Massard and Lange ${ }^{20)}$ expressed the rate of gas entrainment $V_{g}$ by Eq. (25), assuming that gas enclosed between a symmetrical sinusoidal stream surface and a cylindrical plane circumscribed with such a sinusoidal surface is entrained.

$$
V_{g} / V_{l}=2 \varepsilon / a+\varepsilon^{2} /\left(2 a^{2}\right)
$$

Sande and Smith ${ }^{17}$ ) also described the value of $V_{g}$ by Eq. (26), where $A_{3}$ and $\theta$ represent the constant and the angle of impact (angle between stream and liquid surface), taking the kinetic energy of teeming stream into account.

$$
V_{g}=A_{3} D_{0}^{3 / 2} U_{0}^{2} z^{1 / 3} / \sin \theta
$$

In the present work, the gas entrainment rates are proposed by the model in which the cavity formation by the collision between pouring stream and liquid pool, and the disturbance on the stream surface are taken into account.

\section{Experimental Method}

For the measurement of the rate of gas entrainment by water, ethanol and glycerin aqua solution, the apparatus shown by Fig. 12(A) was used under the constant teeming velocity and also Fig. 12(B) was used under changing teeming velocity. In both cases, stream $(\mathrm{h})$ is shielded with gum bellows (d) that allow the teeming height $z$ to be controlled. For the study on the teeming of liquid tin, a series of double induction coils as shown by Fig. 12(G) was used. Tin of about $700 \mathrm{~g}$ charged in upper crucible (e) and about $1000 \mathrm{~g}$ in lower crucible (f) were simultaneously melted under argon atmosphere, and the temperature
(A)

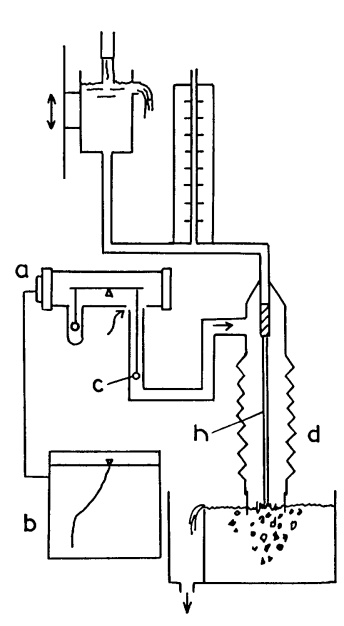

(B)

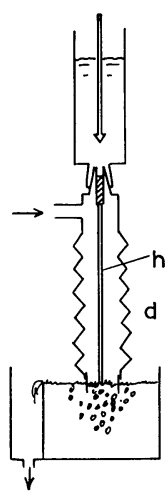

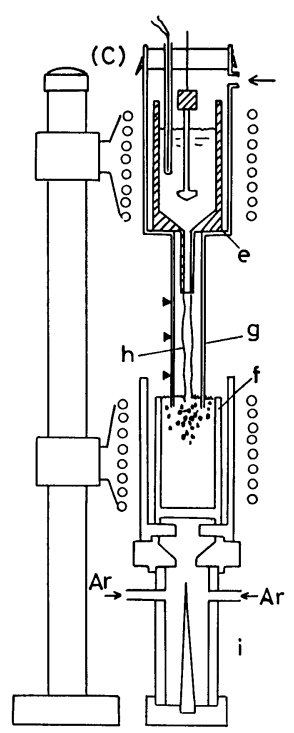

Fig. 12. Experimental apparatus for the measurement of gas entrainment. was maintained at $350{ }^{\circ} \mathrm{C}$ for ten minutes. Then, by raising the stopper of the upper crucible, liquid tin was teemed into the lower crucible where liquid tin overflowed into the mold (i) set below the lower crucible in order to keep the teeming height $z$ at constant level. In this case, the transparent quartz tube (g) was used for the protection of stream from air. By the temperature measurement at the position of mark on quartz tube, it was confirmed that the temperature change of the atmosphere was negligible.

In every case, the gas entrainment rates were measured by recording the downward force on the glass ball (c) hanging from the electric balance (a) due to the flow of the air supplied from outside.

Experimental error in the present work was investigated by counting the number of bubbles on the surface of pool in the inside and the outside of seal pipe by taking several still photographs during teeming. When teeming height is low, the most of bubbles entrained go out from the seal pipe. On the other hand, for higher teeming height such as $z / D_{0}>45$, $4 \sim 6 \%$ of entrained bubbles return again to the inside of seal pipe, resulting in the error of measurement.

\section{Experimental Results and Discussion}

\section{Gas Entrainment Rate $\mathbf{V}_{\boldsymbol{g}}$}

At first, the gas entrainment rates of water were measured under the constant teeming rate. The experimental results for nozzle diameter $D_{0}=0.8 \mathrm{~cm}$ are shown in Fig. 13, which represents that the gas entrainment occurs at more than certain teeming height, above which the entrainment rate increases with increasing teeming height, and also with increasing teeming rate. The same behavior was also found for nozzle diameters $D_{0}=0.6$ and $1.0 \mathrm{~cm}$. The values of $V_{g} / V_{l}$ obtained from those experimental results do not satisfy Eq. (25) by Massard and Lange ${ }^{20)}$ nor Eq. (26) by Sande and Smith. ${ }^{17)}$ This is probably because, in the model by Massard and Lange, the bath motion such as the cavity created by the collision of stream onto the bath is ignored, and in the study of Sande 


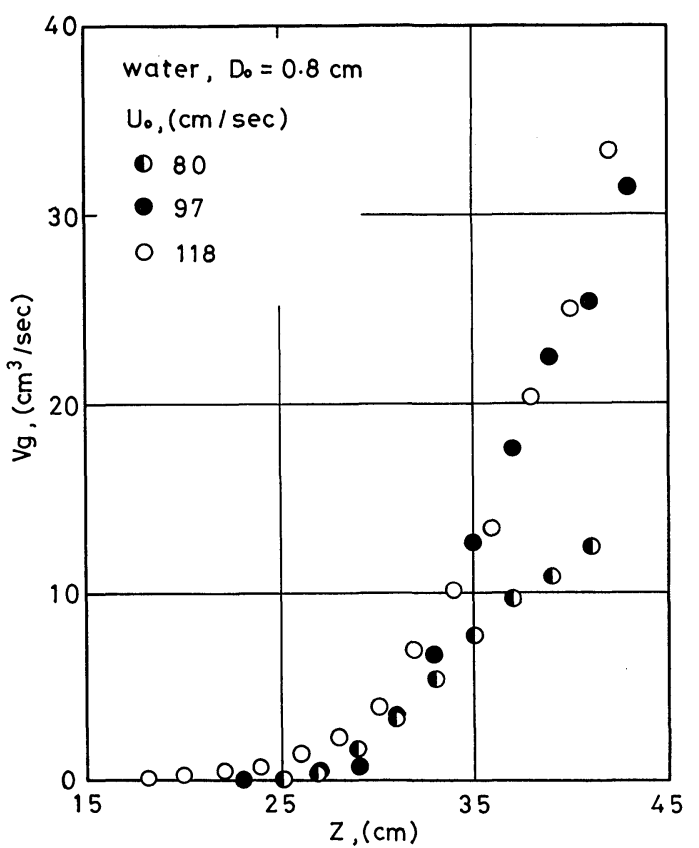

Fig. 13. Dependence of the rate of gas entrainment on the stream length.

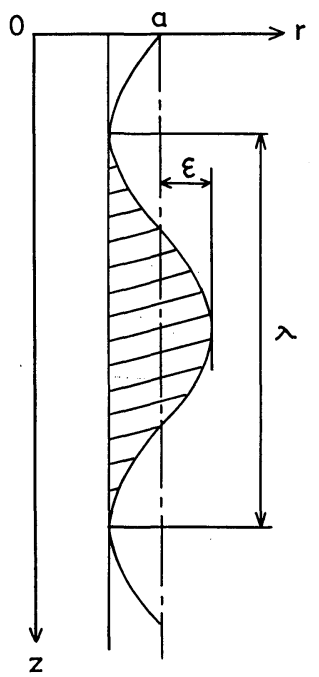

Fig. 14.

Model for the disturbance on the surface of liquid stream.

and Smith, the effect of the disturbance of the stream surface on the gas entrainment rate is neglected although the kinetic energy is taken into account.

In the present work, it is assumed that only kinetic energy of protuberant portion shown in Fig. 14 (oblique line part) controls the gas entrainment. Describing the radius of jet at $z$ by $a+\varepsilon \cos k z$, the mass of protuberant portion, $m$, is given by $\mathrm{Eq}$. (27).

$$
\begin{aligned}
m & =\pi \rho \int_{0}^{\lambda}\left\{(a+\varepsilon \cos k z)^{2}-(a-\varepsilon)^{2}\right\} d z \\
& =\pi \rho \lambda\left(2 a \varepsilon-\varepsilon^{2} / 2\right) \quad \ldots \ldots \ldots \ldots \ldots \ldots \ldots \ldots \ldots \ldots \ldots
\end{aligned}
$$

The kinetic energy is given by $E_{1}=(1 / 2) m U^{2}$. Here, using $a, \xi, U, \varepsilon_{0}$, and $\lambda$ by Eqs. (9), (10), (11), (24) and (5), and calculating $\varepsilon$ from Eq. (17) simplified by using $\left\{(4 / 3)\left(\rho_{g} / \rho\right)+W e^{-1}\right\}^{1 / 2} \simeq 0.5 W e^{-0.35}$, the value of $E_{1}$ can be obtained from Eq. (27).

On the other hand, the cavity formation at the collision area between the stream and the water pool

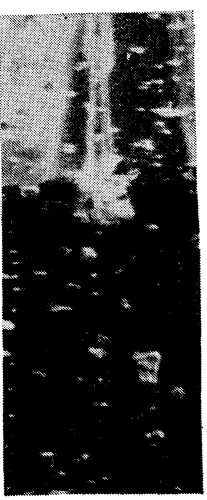

a

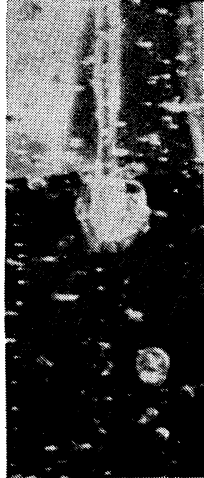

$\mathrm{b}$

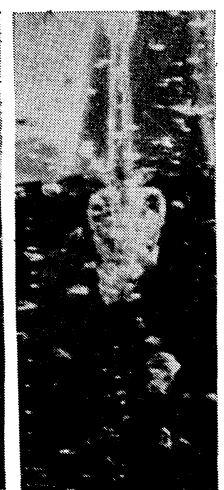

C

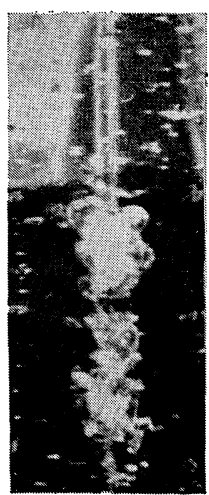

d
(a) $0.7 \times 10^{-3} \mathrm{sec}$
(c) $2.2 \times 10^{-3} \mathrm{sec}$
(b) $1.3 \times 10^{-3} \mathrm{sec}$
(d) $5.5 \times 10^{-3} \mathrm{sec}$

Photo. 1. Behavior of cavity produced by pouring water stream.

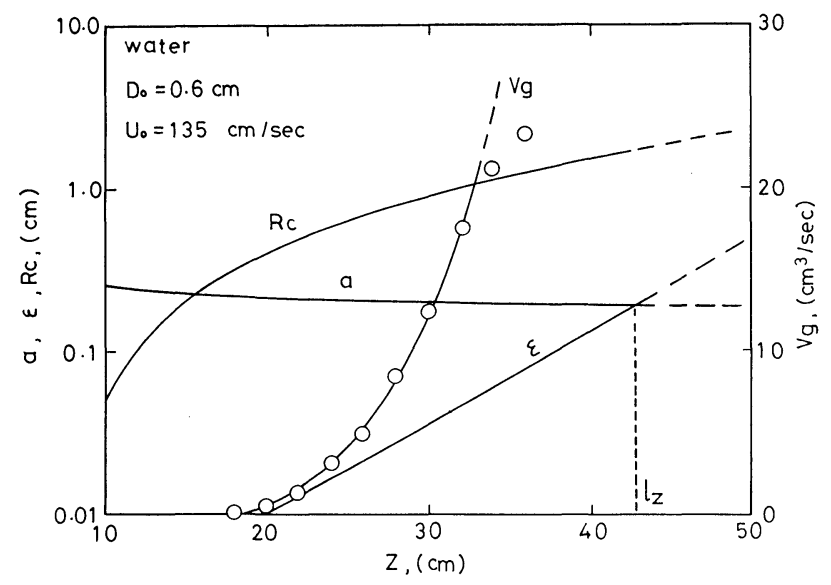

Fig. 15. Dependence of $a, \varepsilon, R c$ and $V_{g}$ on the stream length.

surface was found as shown in Photo. 1. The cavity and gas bubbles are found in the dark part of the photographs. As seen in those photographs the cavity appears at Photo. 1(a), and it becomes almost a sphere at Photo. 1(b). Then it breaks gradually into many small bubbles as found in Photos. 1 (c) and (d).

Referring to this observation, the experimental results were analyzed by taking the cavity radius $R_{c}$ into consideration. Now, using the differential equation which was obtained by Sande et al. ${ }^{21)}$ for the cavity produced by the collision between the bath surface and a water droplet, $R_{c}$ can be represented by Eq. (28).

$$
R_{c}=\left\{\left(1.14+0.00089 E_{1}\right)^{1 / 2}-1.067\right\}^{1 / 2}
$$

As an example, the values $a, \varepsilon, V_{g}$ and $R_{c}$ calculated from Eq. (28) are plotted against $z$ in Fig. 15, which shows that the gas entrainment occurs near the point where the stream radius almost becomes the same as the cavity radius, that is, $a \approx R_{c}$. Figure 16 gives the relationship between $\left(R_{c}-a\right) / a_{0}$ and $V_{g} / V_{l}$. It is found that gas entrainment rate is represented by Eq. (29) for all experiments under different teeming rates and nozzle diameters.

$$
V_{g} / V_{l}=0.02\left\{\left(R_{c}-a\right) / a_{0}\right\}^{3}
$$




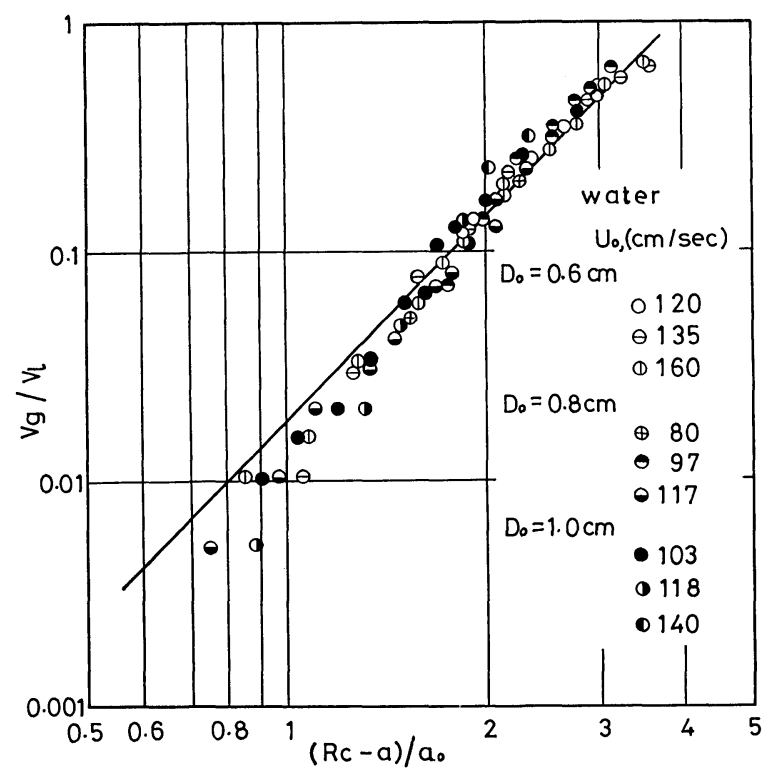

Fig. 16. Dimensionless correlation between $\log \left(V_{g} / V_{l}\right)$ and $\log \left\{\left(R_{c}-a\right) / a_{0}\right\}$.

Next, by using the apparatus shown in Fig. 12(B), the gas entrainment rates were measured under the condition that the teeming rate depends on time. The typical results of water stream of $D_{0}=1.0 \mathrm{~cm}$ are shown in Fig. 17. It is recognized that, in all cases of the teeming height, the gas entrainment rates decrease with increasing teeming time because of the decrease of flow velocity at the nozzle exit. Now, calculating $U_{0}, \varepsilon_{0}, a, \varepsilon$ and $R_{c}$ for the experimental results from Eqs. (20), (24), (9), (17) and (28) individually, the values of $V_{g} / V_{l}$ are obtained by Eq. (29). The experimental values of $V_{g} / V_{l}$ are plotted against $\left(R_{c}-a\right) / a_{0}$ in Fig. 18, which shows those values approximately agree well with the relationship shown by Eq. (29). Accordingly it is reasonable to consider that Eq. (29) obtained under the constant teeming rate is also applicable for this system. Furthermore, it is clear from Figs. 19 to 21 that Eq. (29) represents the experimental results for glycerin aqua solution, ethanol and liquid tin measured by the apparatus shown in Fig. 12(C). In some cases, the values of $V_{g} / V_{l}$ at the initial stage of those experiments deviate remarkably from the relation of Eq. (29) and, after several seconds, they gradually approach the line represented by Eq. (29). This appears to have resulted from the strong surface disturbance due to the initial unstable condition of the stream.

\section{Diameters of the Entrained Gas Bubbles}

The diameters of gas bubbles entrained, $d_{B}$, were measured photographically. It was observed that the values of $d_{B}$ did not depend on the gas entrainment rate, but it increased with increasing nozzle diameter. This agrees well with the results by Lin and Donnelly ${ }^{22}$ ) who described that the diameter of stream $D$ at the bath surface determined the value of $d_{B}$. According to the present results, however, it is found that the value of $d_{B}$ depends on the kind of fluid even under the same nozzle diameter. Now, taking the physical properties of fluid into account, the values

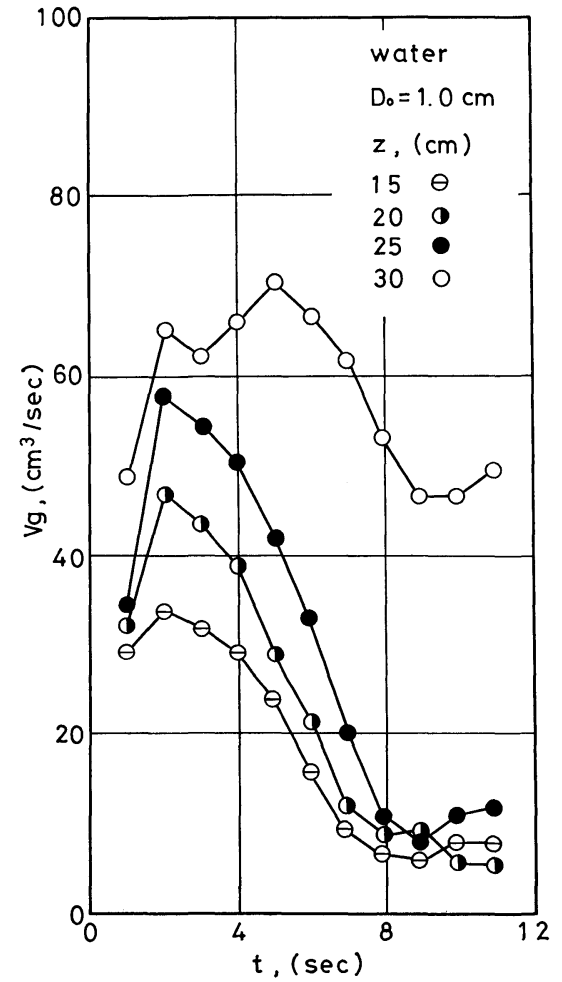

Fig. 17. Time dependence of $V_{g}$ for teeming from the batch type tank.

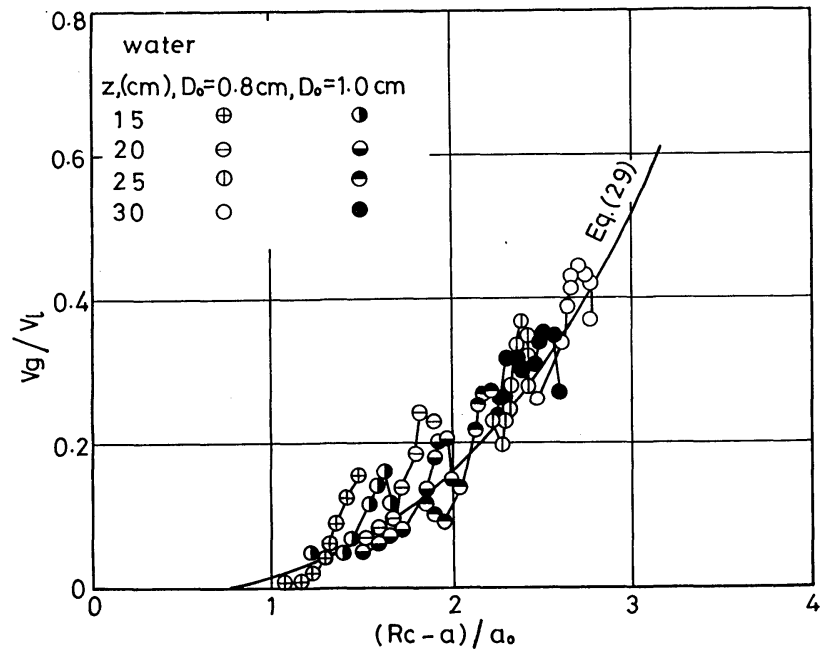

Fig. 18. Dimensionless correlation between $V_{g} / V_{l}$ and $\left(R_{c}-a\right) / a_{0}$ for water stream. Curve is represented by Eq. (29).

of $d_{B}$ could be expressed by Eq. (30) as shown in Fig. 22.

$$
\begin{aligned}
d_{B} & =0.004 \rho^{3 / 4} \cdot g^{1 / 2} \cdot D^{3 / 4} /\left(\mu^{1 / 2} \cdot \sigma^{1 / 4}\right) \\
& =0.004 D^{-1 / 2} \cdot R e^{1 / 2} \cdot F r^{-1 / 2} W e^{1 / 4}
\end{aligned}
$$

Accordingly, the value of $d_{B}$ for each fluid is proportional to $D^{3 / 4}$ and the liquid iron stream of the diameter $D=5 \mathrm{~cm}$ at the bath surface may entrain the gas bubbles of $d_{B}=1.3 \mathrm{~cm}$.

\section{Conclusion}

In order to study the gas entrainment of liquid steel during teeming, the model experiments were 


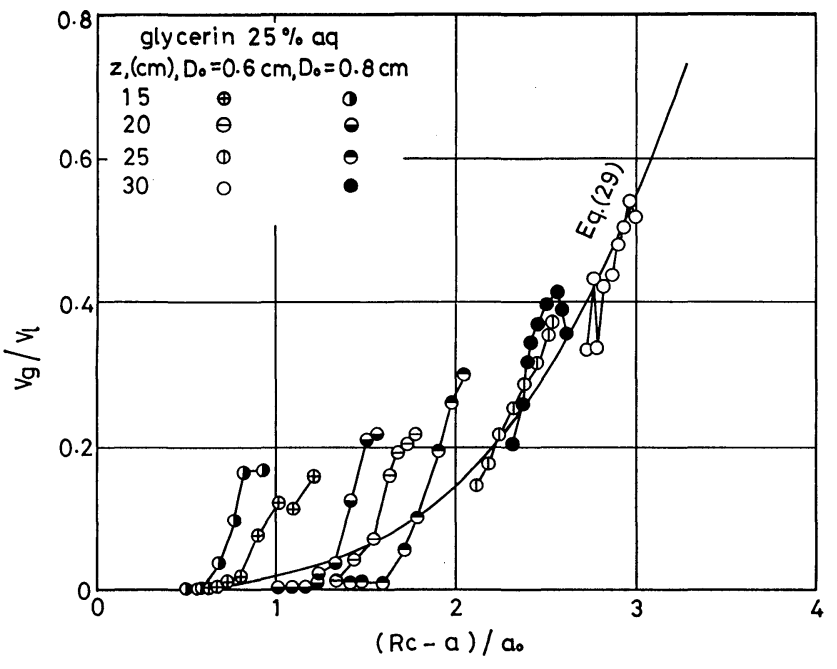

Fig. 19. Dimensionless correlation between $V_{g} / V_{l}$ and $\left(R_{c}-a\right) / a_{0}$ for glycerin $25 \%$ aqua solution. Curve is represented by Eq. (29).

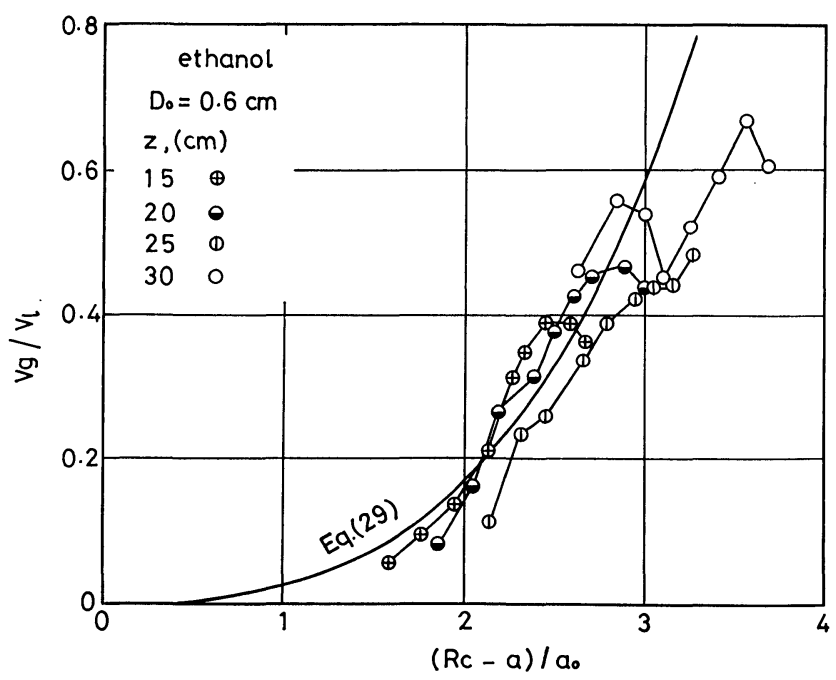

Fig. 20. Dimensionless correlation between $V_{g} / V_{l}$ and $\left(R_{c}-a\right) / a_{0}$ for ethanol stream. Curve is represented by Eq. (29).

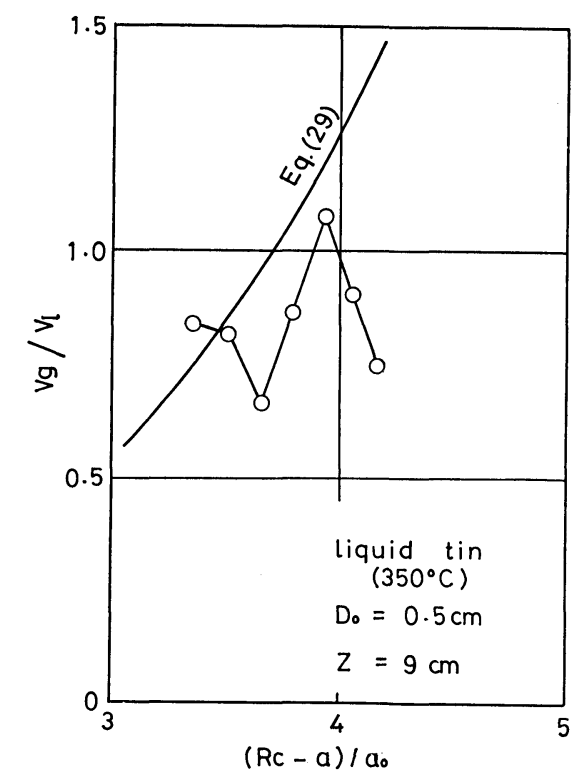

Fig. 21. Dimensionless correlation between $V_{g} / V_{l}$ and $\left(R_{c}-a\right) / a_{0}$ for liquid tin.

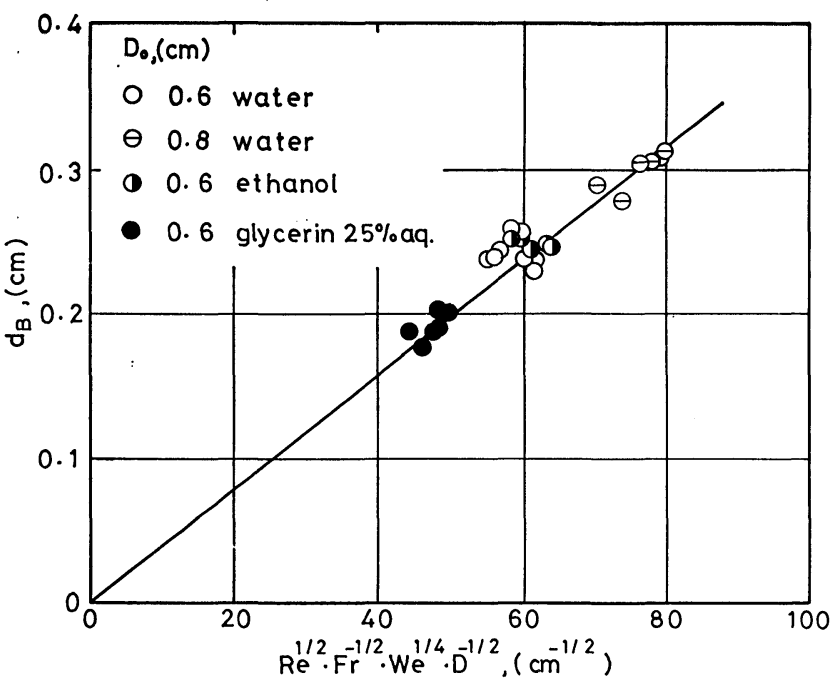

Fig. 22. Relation between the diameter of entrained bubble and $\operatorname{Re}^{1 / 2} \mathrm{Fr}^{-1 / 2} \mathrm{We}^{1 / 4} \mathrm{D}^{-1 / 2}$.

made by using water, ethanol, glycerin aqua solution and liquid tin.

(1) The breakup length of stream, $l_{z}$, depends on the nozzle geometry, teeming rate and the motion of fluid in the tank, and can be expressed by the following relationship.

$$
l_{z} / D_{0}=A W e^{2 / 5} \mathrm{Fr}^{-1 / 7}
$$

(2) The initial amplitude of surface disturbances, $\varepsilon_{0}$, calculated by the value of $l_{z}$ can be represented by the following equation.

$$
\ln \left(a_{0} / \varepsilon_{0}\right)=44.3\left(l_{0} / D_{0}\right)^{-0.75} R e^{-0.1}
$$

(3) The gas entrainment rate increases with increasing teeming height and increasing teeming rate.

(4) The gas entrainment occurs due to the disturbance of stream surface and the entrainment rate depends on the cavity radius created by the collision between the stream and the bath surface. The values of $V_{g} / V_{l}$ are described in dimensionless expression as follows:

$$
V_{g} / V_{l}=0.02\left\{\left(R_{c}-a\right) / a_{0}\right\}^{3}
$$

(5) Although the diameter of gas bubble entrained is independent of gas entrainment rate, it can be given by the following relation in terms of the physical properties and the stream diameter $(D)$ at the bath surface.

$$
d_{B}=0.004 R e^{1 / 2} F^{-1 / 2} W e^{1 / 4} D^{-1 / 2}
$$

\section{Nomenclature}

$a, a_{0}$ : Radius of liquid stream at $z$ and nozzle exit (cm)

$d_{B}$ : Diameter of gas bubble entrained (cm)

$D$ : Diameter of stream at the bath surface $(\mathrm{cm})$

$D_{0}$ : Diameter of nozzle $(\mathrm{cm})$

$E_{1}$ : Kinetic energy (erg)

Fr: Froude number $\left(-,=U_{0} /\left(D_{0} g\right)\right)$

$h, h_{i}$ : Depth of fluid in the tank and its initial value $(\mathrm{cm})$

$l_{0}$ : Nozzle length $(\mathrm{cm})$ 
$l_{z}:$ Breakup length of stream $(\mathrm{cm})$

$m$ : Mass of protuberant portion of the stream (g)

$R_{1}:$ Radius of tank (cm)

$R_{c}$ : Radius of cavity (cm)

$R e:$ Reynolds number $\left(-,=\rho D_{0} U_{0} / \mu\right)$

$t$ : Time (sec)

$U, U_{\mathbf{0}}$ : Flow velocities at $z$ and at the nozzle exit $(\mathrm{cm} / \mathrm{sec})$

$V_{g}, V_{l}:$ Gas entrainment rate and volumetric flow rate of stream $\left(\mathrm{cm}^{3} / \mathrm{sec}\right)$

$W e$ : Weber number $\left(-,=U_{0}^{2} \rho D_{0} / \sigma\right)$

$W_{\max }$ : Maximum growth rate of stream disturbance $(1 / \mathrm{sec})$

$z$ : Teeming height $(\mathrm{cm})$

$\varepsilon, \varepsilon_{0}$ : Amplitude of surface disturbance of stream at $z$ and that at nozzle exit $(\mathrm{cm})$

$\lambda$ : Wave length $(\mathrm{cm})$

$\rho, \rho_{g}$ : Density of fluid and gas $\left(\mathrm{g} / \mathrm{cm}^{3}\right)$

$\sigma:$ Surface tension $(\mathrm{dyn} / \mathrm{cm})$

$\mu: \operatorname{Viscosity}(\mathrm{g} /(\mathrm{cm} \mathrm{sec}))$

\section{Acknowledgements}

This work was partly supported by the Grant in Aid of the Ministry of Education, Japan in 1979.

\section{REFERENCES}

1) R. P. Grant and S. Middleman: AIChEJ, 12 (1966), 669.

2) A. G. Merrington and E. G. Richardson: Proc. Phys. Soc., 5 (1947), 59.
3) D. F. Rulland and G. J. Jameson: Chem. Eng. Sci., 25 (1970), 1689.

4) Y. Tanazawa and S. Toyoda: Trans. Japan Soc. Mech. Eng., 20 (1954), 299.

5) L. Rayleich: Proc. London Math. Soc., 10 (1878), 7.

6) G. Weber: Z. Angew. Math. Mech., 11 (1931), 136.

7) V. G. Levich: Physicochemical Hydrodynamics, PrenticeHall, Inc., Englewood Cliffs, N.J., (1962), 626.

8) T. Takahashi and Y. Kitamura: Kagaku Kogaku (Chemical Eng. Japan), 35 (1971), 1229, and 36 (1972), 527.

9) T. A. Engh and K. Larsen: Scand. J. Met., 8 (1976), 161.

10) F. Savart: Ann. Chim., 53 (1883), 337.

11) W. Ohnesorge: Z. Angew. Math. Mech., 16 (1936), 355.

12) P. Massard and K. W. Lange: Arch. Eisenhüttenw., 47 (1976), 595.

13) P. Massard and K. W. Lange: Arch. Eisenhüttenw., 48 (1977), 65.

14) R. L. Duda and J. S. Vrentas: Chem. Eng. Sci., 22 (1976), 855.

15) T. Kurabayashi: Trans. Japan Soc. Mech. Eng., 25 (1959), 1266.

16) A. Ziabicki and R. T. Krozer: Rocniki Chemii, 37 (1963), 113.

17) E. van de Sande and J. M. Smith: Chem. Eng. Sci., 31 (1976), 219.

18) Y. Koike: Tetsu-to-Hagané, 44 (1958), 122.

19) Y. N. Yakovlev: Stahl in USSR, 3 (1973), 480.

20) P. Massard and K. W. Lange: Arch. Eisenhüttenw., 48 (1977), 521.

21) E. van de Sande, J. M. Smith and J.J.J. van Oord: J. Appl. Phys., 45 (1974), 748.

22) T.J. Lin and H. G. Donnelly: AIChEJ, 12 (1966), 563. 